\title{
Disentangling AGN and starburst activities in NGC 6240 from an X-ray perspective
}

\author{
Junfeng Wang \\ Department of Astronomy and Institute of Theoretical Physics and Astrophysics, \\ Xiamen University, Xiamen, Fujian 361005, China \\ email: jfwang@xmu.edu.cn
}

\begin{abstract}
The circum-nuclear region in an active galaxy is often complex with presence of high excitation gas, collimated radio outflow, and star formation activities, besides the actively accreting supermassive black hole. The unique spatial resolving power of Chandra X-ray imaging spectroscopy enables more investigations to disentangle the active galactic nuclei and starburst activities. For galaxies in the throes of a violent merging event such as NGC6240, we were able to resolve the high temperature gas surrounding its binary active black holes and discovered a large scale soft X-ray halo.
\end{abstract}

Keywords. black hole physics, galaxies: active, galaxies: interactions, galaxies: halos, X-rays: ISM

\section{Introduction}

The current picture of galaxy evolution advocates co-evolution of galaxies and their nuclear massive black holes (MBHs), through accretion and merging, which successfully explains much of the observed active galactic nuclei (AGN) statistics and the $M_{\mathrm{BH}}-\sigma$ relation. Quasar pairs (a few kpc-100 kpc separation) and the sub-light-year separation MBHs exemplify the early through late stages of the gravitational interaction. The double active nuclei of few nearby galaxies with disrupted morphology and intense star formation demonstrate the importance of major mergers of equal mass spirals in this evolution, leading to formation of a massive elliptical galaxy. In studies of the hot interstellar medium in galaxies experiencing these key stages, the full exploitation of Chandra imaging potential coupled with its CCD spectroscopy capabilities gives us the opportunity to deeply investigate the physical state of the diffuse X-ray emission at increasing distance from the central AGN, overcoming the contamination from bright point sources.

\section{The infrared luminous galaxy merger NGC 6240}

In the local universe, NGC $6240(z=0.02448 \pm 0.00003)$ is a unique galaxy, in the throes of a violent merging event and on its way to becoming an elliptical galaxy (Engel et al. 2010). It is experiencing intense star formation (e.g., $61 \pm 30 \mathrm{M}_{\odot} \mathrm{yr}^{-1}$ in Yun \& Carilli 2002). With $L_{F I R} \sim 10^{11.8} \mathrm{M}_{\odot}$ just below $10^{12} \mathrm{M}_{\odot}$, the luminosity threshold for the ultraluminous infrared galaxies (ULIRGs), NGC 6240 is expected to become an ULIRG when the galaxies coalesce and a final starburst is triggered (Engel et al. 2010). The Chandra X-ray images Komossa et al. 2003 of the central region of NGC 6240 revealed two gravitationally interacting active supermassive black holes (SMBHs) separated by $\sim 0.7 \mathrm{kpc}$. These sources are characterized by the highly absorbed hard X-ray spectra typical of Compton thick active galactic nuclei (AGNs). They each have an observed luminosity of $L_{2-8 \mathrm{keV}} \sim 10^{42} \mathrm{erg} \mathrm{s}^{-1}$, and show prominent neutral Fe $\mathrm{K} \alpha$ 

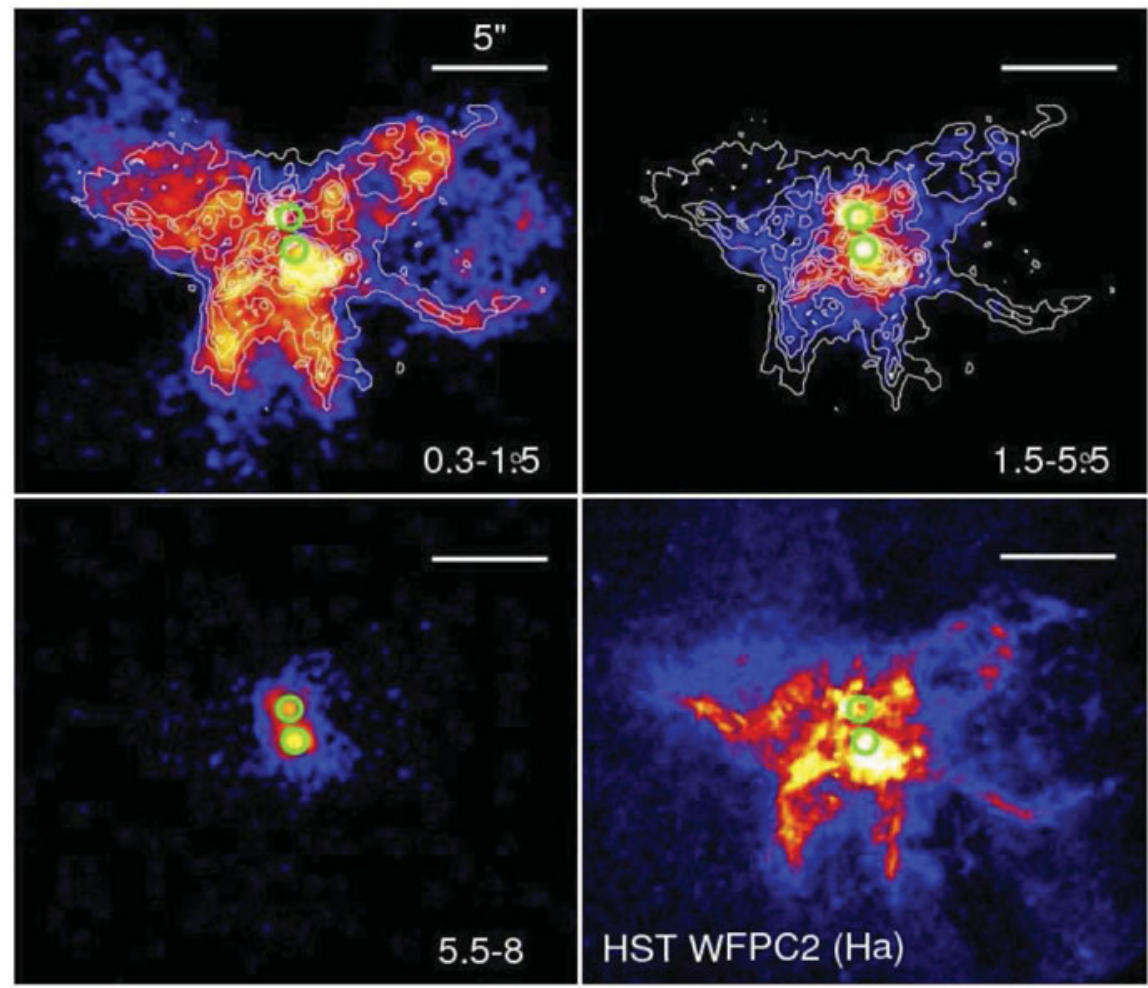

Figure 1. Comparison of multi-band X-ray emission and the HST/WFPC2 $\mathrm{H} \alpha$ image. Top left: $0.3-1.5 \mathrm{keV}$ soft X-ray emission; top right: $1.5-5.5 \mathrm{keV}$ X-ray emission; bottom left: $5.5-8$ $\mathrm{keV}$ X-ray emission, dominated by the two nuclei; bottom right: HST/WFPC2 image of the $\mathrm{H} \alpha$ emitting gas, from which the white contours (shown in the top panels) were generated.

lines at $6.4 \mathrm{keV}$ present in the spectra of both nuclei. Marginal evidence of an hydrogenlike iron line emission was found, which could be associated with a spatially resolved, multi-temperature hot gas outflow, powered by the starburst activity that dominates the 0.5-3 keV X-ray emission $\left(L_{0.1-2 \mathrm{keV}} \sim 10^{43} \mathrm{erg} \mathrm{s}^{-1}\right.$; Huo et al. 2004). XMM-Newton observations (Netzer et al. 2005) resolved the Fe K line complex into three narrow lines, the neutral Fe K $\alpha$, Fe XXV, and a blend of Fe XXVI with Fe K $\beta$. These XMM-Newton data, however, do not have the resolution to resolve spatially the line-emitting regions.

\section{Results}

We report on a recent $150 \mathrm{ks}$ long, sub-arcsecond resolution X-ray image of the nuclear region of the luminous galaxy merger NGC 6240 with Chandra, which resolves the X-ray emission from the pair of active nuclei and the diffuse hot gas in great detail (Figure 1; see Wang et al. 2014). We detect extended hard X-ray emission from $k T \sim 6 \mathrm{keV}(\sim 70$ million $\mathrm{K}$ ) hot gas over a spatial scale of $5 \mathrm{kpc}$, indicating the presence of fast shocks with velocity of $\sim 2000 \mathrm{~km} \mathrm{~s}^{-1}$. For the first time we obtain the spatial distribution of this highly ionized gas emitting $\mathrm{Fe} \mathrm{XXV}$, which shows a remarkable correspondence to the large scale morphology of $\mathrm{H}_{2}(1-0) \mathrm{S}(1)$ line emission and $\mathrm{H} \alpha$ filaments. Propagation of fast shocks originated in the starburst driven wind into the ambient dense gas can account for this morphological correspondence. 
With an observed $L_{0.5-8 \mathrm{keV}}=5.3 \times 10^{41} \mathrm{erg} \mathrm{s}^{-1}$, the diffuse hard X-ray emission is $\sim 100$ times more luminous than that observed in the classic starburst galaxy M82. We estimate its total mass $\left(M_{\mathrm{hot}}=1.8 \times 10^{8} \mathrm{M}_{\odot}\right)$ and thermal energy $\left(E_{\mathrm{th}}=6.5 \times 10^{57}\right.$ erg). The total iron mass in the highly ionized plasma is $M_{\mathrm{Fe}}=4.6 \times 10^{5} \mathrm{M}_{\odot}$. Both the energetics and the iron mass in the hot gas are consistent with the expected injection from the supernovae explosion during the starburst that is commensurate with its high star formation rate. However, a recent $\mathrm{CO}(1-0)$ interferometry observation of NGC 6240 (Feruglio et al. 2013) implies a total kinetic power of the outflowing CO gas $E_{k i n}=$ $3.8 \times 10^{57} \mathrm{erg}$, which leads to the plausible extra heating from the central double AGN.

We also detected an extended hot halo (Nardini et al. 2013), with projected physical size of $\sim 110 \times 80 \mathrm{kpc}$, and a single-component thermal model provides a reasonably good fit to the observed X-ray spectrum. The hot gas has a temperature of $7.5 \mathrm{MK}$ and an estimated total mass of $10^{10} \mathrm{M}_{\odot}$, resulting in an intrinsic $0.4-2.5 \mathrm{keV}$ luminosity of $4 \times 10^{41} \mathrm{erg} \mathrm{s}^{-1}$. The relative abundance of the main alpha-elements with respect to iron is several times the solar value, and nearly constant as well, implying a uniform enrichment by type II supernovae out to the largest scales. Taken as a whole, the observational evidence hints at widespread, enhanced star formation proceeding at steady rate over the entire dynamical timescale (about $200 \mathrm{Myr}$ ). Under favorable conditions, at least a fraction of it might be retained after the merger completion, and evolve into the hot halo of a young elliptical galaxy.

\section{Acknowledgements}

This work was supported by NASA grant GO1-12123X and the National Natural Science Foundation of China under grants 11443003 and 11473021.

\section{References}

Engel, H., Davies, R. I., Genzel, R., Tacconi, L. J., Hicks, E. K. S., Sturm, E., Naab, T., Johansson, P. H., Karl, S. J., Max, C. E., Medling, A., \& van der Werf, P. P. 2010, A\&AA, 524, A56

Feruglio, C., Fiore, F., Maiolino, R., Piconcelli, E., Aussel, H., Elbaz, D., Le Floc'h, E., Sturm, E., Davies, R., \& Cicone, C. 2013, A\&3A, 549, A51

Huo, Z. Y., Xia, X. Y., Xue, S. J., Mao, S., \& Deng, Z. G. 2004, ApJ, 611, 208

Komossa, S., Burwitz, V., Hasinger, G., Predehl, P., Kaastra, J. S., \& Ikebe, Y. 2003, ApJ (Letters), 582, L15

Nardini, E., Wang, J., Fabbiano, G., Elvis, M., Pellegrini, S., Risaliti, G., Karovska, M., \& Zezas, A. $2013, A p J, 765,141$

Netzer, H., Lemze, D., Kaspi, S., George, I. M., Turner, T. J., Lutz, D., Boller, T., \& Chelouche, D. 2005, ApJ, 629, 739

Wang, J., Nardini, E., Fabbiano, G., Karovska, M., Elvis, M., Pellegrini, S., Max, C., Risaliti, G., U, V., \& Zezas, A. 2014, ApJ, 781, 55

Yun, M. S. \& Carilli, C. L. 2002, ApJ, 568, 88 